\title{
Automedicação em idosos da Atenção Básica
}

\author{
Self-medication in elderly primary care
}

\author{
Thalita Cristinny Araujo Silva' ${ }^{1}$ \\ Francisco das Chagas Candeira Mendes Júnior ${ }^{2}$ (i) \\ Jefferson Carlos Araujo Silva ${ }^{3}$ (1)
}

\section{Jeane de Sousa Carvalho ${ }^{4}$ Mara Dayanne Alves Ribeiro 5 (1) Fernando Barcellar Biângulo 6 (1)}

1,4Secretaria Municipal de Saúde de Cocal (Cocal). Piauí, Brasil. cristinny.thalita@gmail.com, jeanesousa123@hotmail.com ${ }^{2}$ Faculdade Maurício de Nassau (Parnaíba). Piauí, Brasil. fcmendesjrfisio@gmail.com ${ }^{3}$ Autor para correspondência. Universidade de Brasília (Brasília). Distrito Federal, Brasil. jeffcasilva@gmail.com ${ }^{5}$ Hospital Regional do Norte de Sobral (Sobral). Ceará, Brasil. mara_dayanne2@hotmail.com 6Universidade de Brasília (Brasília). Distrito Federal, Brasil. fernandobarcellar@gmail.com

\begin{abstract}
RESUMO | OBJETIVO: Descrever a prática da automedicação em idosos atendidos na Atenção Básica. MÉTODO: Estudo transversal, descritivo e exploratório realizado com idosos adscritos na Estratégia Saúde da Família (ESF) Mutirão do município de Cocal, Piauí. Foram incluídos idosos com autonomia física e funcional. Os dados foram coletados por meio da aplicação de um questionário contendo dados sociodemográfico e dados relacionados à prática da automedicação. A análise se deu pela estatística descritiva. RESULTADOS: Um total de 35 idosos integraram a amostra, com média de idade $70( \pm 6,6)$ anos e predominância do sexo feminino $(65,7 \%)$. Verificou-se que $80 \%$ dos participantes referiram fazer uso de medicações de maneira regular, $62,8 \%$ não receberam informações sobre os perigos da automedicação, $68,6 \%$ toma remédios sem prescrição médica e $48,6 \%$ usa medicação indicada por vizinho/amigo/parente. Quadros álgicos seguido da gripe/resfriado foram os motivos mais comuns da automedicação. A orientação farmacêutica $(74,3 \%)$ e ter resolvido o problema de saúde antes da consulta médica $(68,6 \%)$ estão entre as causas que favoreceram a referida pratica. CONCLUSÃO: Os dados sugerem que a automedicação entre os idosos é comum, principalmente em quadros álgicos e resfriados, e que a dificuldade de acesso à consulta médica, orientação farmacêutica e o desconhecimento dos perigos predispõem a esta prática.
\end{abstract}

DESCRITORES: Idoso. Automedicação. Medicamentos sem Prescrição. Atenção Básica.
ABSTRACT | OBJECTIVE: To describe the practice of selfmedication in the elderly assisted in Primary Care. METHOD: Cross-sectional, descriptive, and exploratory study carried out with elderly people enrolled in the Family Health Strategy (FHS) Mutirão in the municipality of Cocal, Piauí, Brazil. Elderly people with physical and functional autonomy were included. The data were collected through the application of a questionnaire containing sociodemographic data and data related to the practice of self-medication. The analysis was carried out using descriptive statistics. RESULTS: A total of 35 elderly were included in the sample, with a mean age of 70 $( \pm 6.6)$ years and a predominance of females $(65.7 \%)$. It was found that $80 \%$ of participants reported using medications regularly, $62.8 \%$ did not receive information about the dangers of self-medication, $68.6 \%$ took medication without a prescription, and $48.6 \%$ used medication indicated by a neighbor/friend/relative. Pain followed by the flu/cold was the most common reason for self-medication. Pharmaceutical counseling (74.3\%) and having solved the health problem before the medical consultation (68.6\%) are among the causes that favored this practice. CONCLUSION: The data suggest that self-medication among the elderly is common, especially in pain and colds, and that the difficulty of access to medical consultation, pharmaceutical guidance, and ignorance of the dangers predispose to this practice.

DESCRIPTORS: Aged. Self-medication. Nonprescription drugs. Primary Health Care. 


\section{Introdução}

O aumento da população de idosos é uma realidade observada no Brasil e no mundo. Segundo dados do Instituto Brasileiro de Geografia e Estatística (IBGE), o Brasil possuía um total de 20.590 .599 idosos no ano de 2010.1 A Pesquisa Nacional por Amostra de Domicílios (PNAD) revelou que em 2013 os idosos representavam $13 \%$ da população total, no ano de 2015 , esse percentual aumentou para $14,3 \%$. No Nordeste, a proporção de idosos representa 13,4\% da população total da região.?

O crescimento do número de idosos implica em uma adequação dos serviços de assistência à saúde em razão do perfil epidemiológico característico dessa parcela da população. Uma maior prevalência de Doenças Crônicas Não Transmissíveis (DCNT), um declínio na capacidade funcional e aumento na morbidade leva o indivíduo idoso ao uso frequente dos serviços de saúde, seja para diagnóstico ou para acompanhamento do tratamento. $\frac{3}{\text { Diante disto, o }}$ Ministério da Saúde (MS) tornou prioritário a atenção à saúde da pessoa idosa e definiu a Atenção Primária à Saúde (APS) como porta de entrada. ${ }^{4}$

A população idosa apresenta diversidade diagnóstica de DCNT e o próprio aumento da longevidade influencia de maneira frequente o uso da terapia farmacológica para tratamento dessas condições. $\mathrm{O}$ medicamento é tido como símbolo de saúde pela população e como bem essencial à saúde, além de importante ferramenta terapêutica. Os medicamentos correspondem aos tratamentos mais utilizados nos serviços de saúde..$^{5}$

As mais variadas disfunções nos sistemas do organismo a que os idosos estão susceptíveis são tratadas com medicamentos, em muitos casos, a pluralidade diagnóstica implica em uma polifarmácia. .6 O medicamento é um importante instrumento coadjuvante na manutenção e recuperação da saúde dos idosos. ${ }^{7}$

A APS é a porta de entrada do Sistema Único de Saúde (SUS) e componente chave deste, executando ações de promoção, proteção e recuperação da saúde. ${ }^{4} A$ integralidade à saúde na APS implica na realização de atividades cabíveis ao seu nível de atenção dentro do SUS, para que haja dispensação de medicamento de qualidade e promoção do seu uso de maneira adequada, contribuindo para uma maior resolutividade na APS.,., 8

A automedicação reflete uma necessidade do homem em participar na sua própria saúde, tanto no que diz respeito à prevenção, como no tratamento da doença. Isso implica que a automedicação é inevitável e intrínseca à natureza humana; constitui-se de uma prática universal, presente nas mais diversas sociedades e culturas. $\stackrel{9}{ }$

Nos indivíduos idosos, a farmacologia apresenta particularidade em razão da redução dos líquidos corporais e da perda de massa muscular. O comprometimento dos metabolismos hepáticos e da função renal dificulta a eliminação dos metabólitos e pode haver acúmulo de substâncias tóxicas e possíveis reações adversas. ${ }^{7}$ Os indivíduos idosos consomem a maior parte da produção mundial de medicamentos e são mais sensíveis aos efeitos destes no organismo. Assim, a prática da automedicação nessa parcela da população merece especial atenção. ${ }^{10}$ Tendo em vista o exposto, o objetivo do presente artigo é descrever a prática da automedicação em idosos atendidos na Atenção Básica.

\section{Métodos}

O estudo possuiu natureza transversal descritivo e exploratório. Foi realizado na Unidade da Estratégia Saúde da Família (ESF) Mutirão, pertencente à Atenção Básica na zona urbana do município de Cocal, Piauí. A referida unidade foi selecionada em função de ser considerada como modelo de gestão a ser seguido pelas demais ESF do munícipio. A autorização para realização da pesquisa foi dada pela Secretaria Municipal de Saúde do Município.

A amostra foi intencional e não probabilística composta por 35 idosos atendidos pela unidade lócus da pesquisa. Os idosos que buscaram atendimento na unidade durante o período de coleta de dados, enquanto aguardavam atendimento, eram convidados a participar do estudo. Os mesmos foram informados acerca dos objetivos da pesquisa e esclarecidos sobre os procedimentos da coleta 
de dados, confirmando sua intenção de participar mediante assinatura do Termo de Consentimento Livre e Esclarecido (TCLE).

Os critérios de inclusão adotados foram: idosos adscritos na área de atuação da ESF escolhida, que realizassem acompanhamento ou que buscaram atendimento durante o período da coleta de dados, com autonomia física e funcional. Foram adotados como critérios de exclusão: idosos com déficit cognitivos que dificultassem o preenchimento dos formulários de pesquisa e/ou idosos que estivessem em processo de transferência de ESF.

A coleta de dados se deu por meio de entrevistas conduzidas em sala reservada da UBS, a fim de preservar a privacidade dos voluntários. Para a coleta de dados os pesquisadores desenvolveram um questionário semiestruturado, contendo questões sobre o perfil sociodemográfico, hábitos de vida (tabagismo, etilismo e sedentarismo) uso de medicação e a prática da automedicação. Com relação às questões sobre sedentarismo, os participantes foram esclarecidos que a condição para não serem enquadrados como sedentários referia-se à prática regular de atividade física, no mínimo três vezes por semana, com duração mínima de 30 minutos. ${ }^{11}$ As questões que investigaram a prática da automedicação nos voluntários possuíam como opções de respostas sim/não.

Questões sobre a prática da automedicação e se os idosos foram informados sobre os perigos da automedicação, se possuíam auxílio para tomar suas medicações habituais e se faziam uso de medicações que não eram receitadas pelo seu médico estavam inclusos no questionário. No instrumento havia ainda questões relacionadas às situações comuns que os levam a fazer uso de medicamentos sem consultar o profissional médico, tais como, dor em diferentes regiões do corpo, gripe/resfriado, febre, entre outros, estavam entre os motivos mais recorrentes.

A coleta dos dados ocorreu entre os meses de agosto a setembro de 2018 e foi realizada por um único avaliador previamente treinado. Os dados foram tabulados no Office Excel versão 2017. Para a análise, foi utilizada estatística descritiva com frequência absoluta para as variáveis qualitativas e média com desvio padrão para as variáveis quantitativas.

Todas as etapas da pesquisa foram desenvolvidas obedecendo as Diretrizes e Normas Regulamentadoras de Pesquisa Envolvendo Seres Humanos do Conselho Nacional de Ética em Pesquisa do MS, obedecendo a resolução 466/2012 do Conselho Nacional de Saúde (CNS). Os procedimentos referentes à coleta e análise dos dadosocorreu somente após aprovação do Comitê de Ética em Pesquisa (CEP) do Instituto de Teologia Aplicada (INTA), CAAE: 92010218.3.0000.8133, com o número de parecer: 2.743.967.

\section{Resultados}

O total de 35 idosos que compuseram a amostra apresentou média de idade de $70( \pm 6,6)$ anos. Os dados referentes à caracterização sociodemográfi$c a$, estilo de vida e presença de DCNT encontramse na tabela 1 , onde observa-se predominância do sexo feminino $23(65,71 \%)$, cor da pele branca 18 $(51,4 \%)$, analfabetos $20(57,1 \%)$, aposentados 24 (68,6\%), estado civil casados $21(60,0 \%)$, residindo com o cônjuge $21(60,0 \%)$ e com renda de um salário mínimo 22 (62,8\%). No que tange ao estilo de vida $4(11,4 \%)$ eram etilistas, $8(22,8 \%)$ tabagista e $21(60,0 \%)$ sedentários. A doença mais frequente foi a cardíaca 19 (54,3\%). 
Tabela 1. Distribuição de acordo com a caracterização sociodemográfica, hábitos de vida e presença de doenças crônicas não transmissíveis em idosos da Atenção Básica, ESF Mutirão, Cocal, Piauí, 2018

\begin{tabular}{|c|c|c|}
\hline Variável & $\mathbf{n}$ & $\%$ \\
\hline \multicolumn{3}{|l|}{ Cor da pele } \\
\hline Branco & 18 & 51,4 \\
\hline Pardo & 11 & 31,4 \\
\hline Preto & 6 & 17,1 \\
\hline \multicolumn{3}{|l|}{ Escolaridade } \\
\hline Analfabeto & 20 & 57,1 \\
\hline Ens. Fund. Incompleto. & 8 & 22,8 \\
\hline Ens. Fund. Completo. & 5 & 14,3 \\
\hline Ens. Méd. Incompleto. & 0 & 0,0 \\
\hline Ens. Méd. Completo. & 1 & 2,8 \\
\hline Ens. Superior & 1 & 2,8 \\
\hline \multicolumn{3}{|l|}{ Profissão } \\
\hline Aposentado & 24 & 68,6 \\
\hline Agricultor & 9 & 25,7 \\
\hline Comerciante & 2 & 5,7 \\
\hline \multicolumn{3}{|l|}{ Estado civil } \\
\hline Casado & 21 & 60,0 \\
\hline Solteiro & 5 & 14,3 \\
\hline Viúvo & 8 & 22,8 \\
\hline Divorciado & 1 & 2,8 \\
\hline \multicolumn{3}{|l|}{ Com quem mora } \\
\hline Cônjuge & 21 & 60,0 \\
\hline Sozinho & 4 & 11,4 \\
\hline Familiar & 10 & 28,6 \\
\hline \multicolumn{3}{|l|}{ Renda } \\
\hline < 1 salário mínimo & 5 & 14,3 \\
\hline 1 salário mínimo & 22 & 62,8 \\
\hline 2 salários mínimos & 6 & 17,1 \\
\hline > 2 salários mínimos & 2 & 5,7 \\
\hline \multicolumn{3}{|l|}{ Hábitos de vida } \\
\hline Etilismo & 4 & 11,4 \\
\hline Tabagismo & 8 & 22,8 \\
\hline Sedentarismo & 21 & 60,0 \\
\hline \multicolumn{3}{|c|}{ Doenças Crônicas Não transmissíveis } \\
\hline Cardíacas & 19 & 54,3 \\
\hline Metabólicas & 7 & 20,0 \\
\hline Neurológicas & 1 & 2,8 \\
\hline Reumatológicas & 1 & 2,8 \\
\hline Osteomioarticulares & 1 & 2,8 \\
\hline Oncológicas & 1 & 2,8 \\
\hline
\end{tabular}

As informações relacionadas às classes de medicamentos utilizadas pelos idosos avaliados estão no gráfico 1 , no qual é verificado maior percentual do uso de anti-hipertensivos (54,28\%), seguido de antidiabéticos (25,72\%).

Gráfico 1. Classes de medicamentos usados por idosos da atenção básica, ESF Mutirão, Cocal, Piauí, 2018

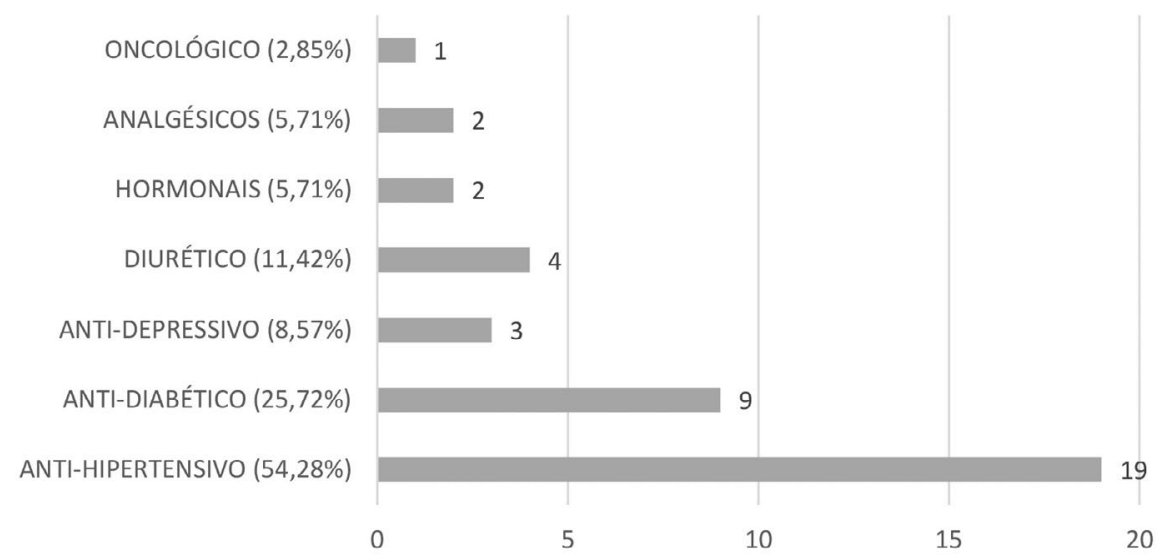


Os dados a cerca da prática da automedicação nos voluntários estão na tabela 2, onde pode ser observado que a maioria, 22 (62,8\%), não foi informada sobre os perigos de fazer uso de medicação sem consultar um profissional médico, 21 (60\%) idosos fazem uso de mais de uma medicação por dia e 24 (68,6\%) tomam medicamentos que não são receitados pelo médico.

Tabela 2. Características da prática da automedicação entre idosos da atenção básica, ESF Mutirão, Cocal, Piauí, 2018

\begin{tabular}{|c|c|c|c|c|}
\hline \multirow[t]{2}{*}{ Questões } & \multicolumn{2}{|c|}{ Sim } & \multicolumn{2}{|c|}{ Não } \\
\hline & $\mathrm{n}$ & $\%$ & $\mathrm{n}$ & $\%$ \\
\hline Você faz uso de medicação regularmente? & 28 & 80 & 8 & 22,8 \\
\hline $\begin{array}{l}\text { Você já foi informado sobre os perigos e danos de tomar medicação sem } \\
\text { conhecimento do seu médico? }\end{array}$ & 13 & 37,1 & 22 & 62,8 \\
\hline $\begin{array}{l}\text { Você possui alguém (esposo/filho/parente/cuidador) que lhe auxilie para tomar } \\
\text { as medicações? }\end{array}$ & 9 & 27,7 & 26 & 74,3 \\
\hline Você costuma lembrar o horário das medicações com precisão? & 24 & 68,6 & 11 & 31,4 \\
\hline Você toma mais de uma medicação por dia? & 21 & 60,0 & 14 & 40,0 \\
\hline Você já se confundiu e trocou o horário das medicações? & 15 & 42,8 & 20 & 57,1 \\
\hline Você sempre vai às consultas com seu médico na UBS? & 17 & 48,6 & 18 & 51,4 \\
\hline Você toma remédios que não sejam receitados pelo seu médico? & 24 & 68,6 & 11 & 31,4 \\
\hline $\begin{array}{l}\text { Você sente algum efeito colateral após tomar a medicação que não é receitada } \\
\text { pelo seu médico? }\end{array}$ & 8 & 22,8 & 27 & 77,1 \\
\hline Você para de fazer uso da medicação sem consultar o seu médico? & 17 & 48,6 & 18 & 51,4 \\
\hline Você já tomou alguma medicação indicada por vizinho/amigo/parente? & 17 & 48,6 & 18 & 51,4 \\
\hline Você costuma fazer uso de chás/garrafadas medicinais como remédio? & 23 & 65,7 & 12 & 34,3 \\
\hline $\begin{array}{l}\text { Você já utilizou receitas emitidas anteriormente pelo seu médico para comprar } \\
\text { medicação? }\end{array}$ & 18 & 51,4 & 17 & 48,6 \\
\hline $\begin{array}{l}\text { Antes de se automedicar, você procurou informações sobre o medicamento que } \\
\text { fez uso? }\end{array}$ & 11 & 31,4 & 24 & 68,6 \\
\hline $\begin{array}{l}\text { Quando você se automedicou estava fazendo uso de medicação receitada pelo } \\
\text { seu médico? }\end{array}$ & 24 & 68,6 & 11 & 31,4 \\
\hline
\end{tabular}

Os dados referentes aos motivos pelos quais os voluntários praticam a automedicação estão dispostos no gráfico 2. Vale destacar que a dor de garganta se sobressaiu, 19 (54,28\%), seguido da dor de cabeça, 17 (48,57\%) e dor óssea, 15 (42,85\%).

Gráfico 2. Avaliação dos motivos pelos quais idosos da atenção básica praticam a automedicação, ESF Mutirão, SUPRIMIDO, 2018

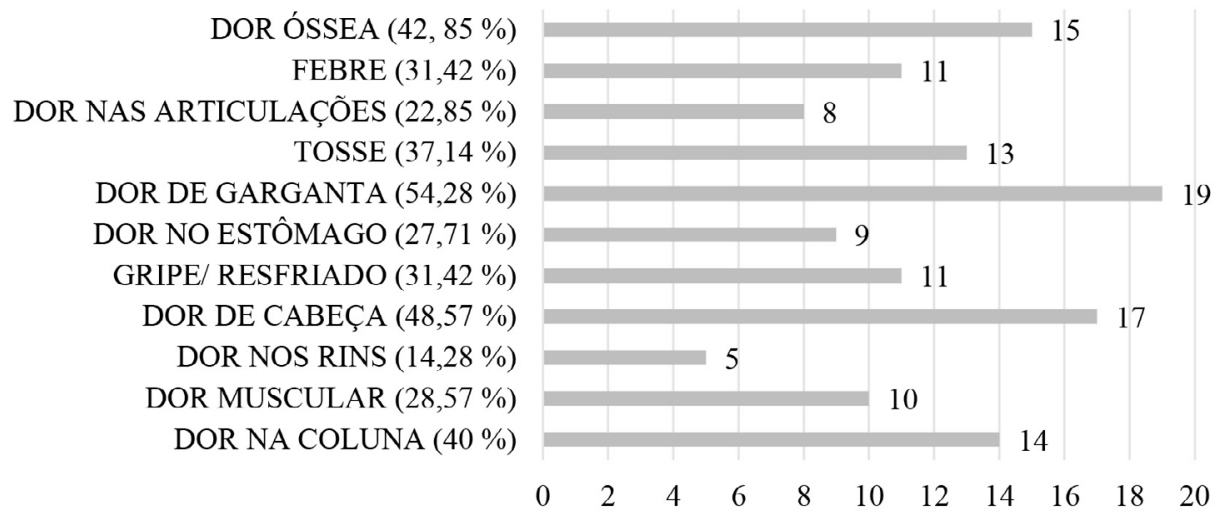


As informações sobre os motivos pelo qual praticavam a automedicação encontram-se na tabela 3, onde verifica-se que orientação farmacêutica foi o motivo citado por $26(74,3 \%)$ dos voluntários, seguido da dificuldade de acesso ao profissional médico, mencionado por $17(48,6 \%)$ dos idosos.

Tabela 3. Motivos para a prática da automedicação entre idosos da atenção básica, ESF Mutirão, Cocal, Piauí, 2018

\begin{tabular}{lcc}
\hline Motivos & $\mathbf{n}$ & $\%$ \\
\hline Dificuldade de acesso ao médico & 17 & 48,6 \\
Falta de tempo para ir ao médico & 8 & 22,8 \\
Orientação de alguém que relatou sintomas parecidos aos seus & 6 & 17,1 \\
Orientação farmacêutica & 26 & 74,3 \\
Ter sido receitado anteriormente pelo médico para os mesmos sintomas & 3,6 \\
Pesquisa na internet ou outro meio de informação & 1 & 2,8 \\
Propaganda em rádio, TV, jornais e/ou revistas & 5 & 14,3 \\
Remédio mais barato do que o receitado pelo médico & 7 & 20,0 \\
Já resolveu o problema de saúde anteriormente, sem consultar ao médico & 24 & 68,6 \\
\hline
\end{tabular}

\section{Discussão}

O presente artigo procurou identificar o perfil da automedicação em idosos atendidos na rede básica de atenção à saúde; para tanto, investigou a presença de DCNT, os medicamentos consumidos habitualmente, informações sobre a prática da automedicação, as situações e os motivos que os levavam a recorrer a tal prática.

A amostra era em sua maioria do sexo feminino, aposentados e de cor branca, esses dados são semelhantes ao de um estudo ${ }^{12}$ desenvolvido em uma UBS de São Luís-MA, que procurou identificar a prevalência, os fatores determinantes e os principais fármacos consumidos sem prescrição entre os idosos da UBS. Os autores concluíram que a automedicação tem alta prevalência entre os idosos. Os analgésicos, devido à facilidade de acesso, foram a classe de medicamentos mais consumida. Em nosso estudo os anti-hipertensivos foram os mais consumidos, acredita-se que tal resultado decorre da DCNT mais frequente, serem as cardíacas, dentre elas a Hipertensão Arterial Sistêmica (HAS).

Estudo desenvolvido em Manaus-AM identificou a prática da automedicação em 75\% dos voluntários, onde 95\% da amostra relatou ser fisicamente ativa e 67,5\% afirmaram possuir HAS, o medicamento sem prescrição médica mais utilizado foi a Dipirona para tratar dor de cabeça e a maioria possuía ensino fundamental incompleto. ${ }^{13} \mathrm{Em}$ contrapartida, no presente estudo, 60\% referiram ser sedentários, as DCNT cardíacas se sobressaíram, seguido das metabólicas, e a maioria dos voluntários eram analfabetos. Não foi investigado qual medicamento sem prescrição médica foi recorrente na presente pesquisa, no entanto, o motivo mais frequente que os levavam a recorrer a tal prática foi a dor de garganta, seguido da dor de cabeça e dor óssea.

A administração de medicamentos em idosos carece de atenção devido à polifarmácia e às mudanças fisiológicas do organismo do idoso, que alteram o processo de absorção dos fármacos. O quadro álgico favorece a prática da automedicação devido à facilidade de acesso aos medicamentos analgésicos. ${ }^{14} \mathrm{~A}$ dor, fosse ela de garganta, cabeça, óssea, coluna, estômago, entre outras, foi o quadro que mais levou os idosos do presente estudo a recorrerem à prática da automedicação. Uma pesquisa ${ }^{15}$ de base populacional desenvolvida em Goiânia-GO avaliou o padrão de consumo de medicamentos de 934 idosos; a maioria pertencia ao sexo feminino, com média de idade de 71,9 anos. Os analgésicos foram consumidos por 9,1\% dos voluntários, onde os autores relatam que o consumo de analgésicos por idosos ocupa lugar de destaque em razão do tratamento da dor e inflamação, situações comuns nessa faixa etária. 
A pluralidade diagnóstica a que o indivíduo idoso está sujeito favorece a polifarmácia e, consequentemente, a automedicação. ${ }^{16} \mathrm{~A}$ maioria dos voluntários do presente estudo referiram fazer uso de medicações de maneira regular, já confundiram os horários das medicações e tomam remédios que não são receitados pelo seu médico. Uma pesquisa ${ }^{17}$ utilizou o instrumento para a Medida de Adesão ao Tratamento (MAT) no intuito de avaliar a adesão à terapêutica medicamentosa de 308 idosos da atenção básica, onde a maioria dos voluntários referiram nunca serem descuidados com os horários da tomada das medicações e nunca deixaram de tomar medicamentos por alguma outra razão que não fosse indicação médica. Os autores concluíram que os idosos possuem adequado nível de adesão à terapêutica medicamentosa e que os profissionais de saúde devem alertar os idosos para os perigos da prática da automedicação.

Em uma UBS de Minas Gerais, 50 idosos referiram que recorrem à prática da automedicação pelo quadro de dor de cabeça, seguido por gripe, e o fato de possuírem o medicamento em casa foi a justificativa mais frequente para a automedicação, onde $88 \%$ dos voluntários afirmaram realizar tal prática mais de 10 vezes ao mês. Os autores concluíram que a prática da automedicação está intimamente relacionada à falta de informações e que a equipe de enfermagem tem papel de educar e informar a população para a sua prevenção. $\frac{18}{} \mathrm{Em}$ nosso estudo a dor de cabeça foi a segunda causa mais frequente dentre os motivos que levaram os voluntários a recorrerem a automedicação e a maioria afirmou que não recebeu informações prévias sobre os riscos de tomar medicação sem conhecimento do seu médico. Mais uma vez, cabe chamar à atenção para a educação em saúde dos idosos quanto aos riscos de se automedicarem e a prevenção dos eventos que advém com sua prática. $\frac{19}{}$

A automedicação em idosos no presente estudo foi propiciada por questões como orientação farmacêutica, resolução da condição sem consulta médica prévia e dificuldade de acesso ao profissional médico, respectivamente nessa ordem. Em um grupo de idosas do Distrito Federal, 30,8\% afirmaram que faziam uso de um ou mais produtos sem prescrição médica e a maioria possuía baixa escolaridade. Os autores destacam que a utilização de medicamentos sem indicação médica pode aumentar o número de medicamentos utilizados pelas idosas e o mascaramento dos sintomas, além das interações medicamentosas e possíveis efeitos adversos.
O estudo afirma que as medidas de atenção farmacêutica devem ser implementadas de maneira eficiente para prevenir a prática da automedicação. $\underline{20}$

Uma pesquisa ${ }^{21}$ desenvolvida com 170 idosos em Belo Horizonte, em sua maioria do sexo feminino, procurou determinar o perfil dos medicamentos utilizados por automedicação nestes indivíduos, 80,6\% afirmaram que praticam a automedicação, os relaxantes musculares e os anti-inflamatórios não esteroidais - AINES, foram os mais frequentes. A pesquisa alerta que o risco de interação medicamentosa é alto, diante do amplo uso de medicamentos de venda livre, além do surgimento de eventos adversos em idosos.

No presente estudo, sete diferentes classes de medicamentos foram identificadas, os anti-hipertensivos e os antidiabéticos foram os mais frequentes em razão das DCNT cardíacas e metabólicas, como HAS e Diabetes Mellitus (DM), respectivamente, serem as mais prevalentes na amostra. Os analgésicos foram responsáveis por $5,71 \%$ da classe de medicamentos de uso contínuo referidos pelos voluntários. No entanto, o quadro álgico, em diferentes regiões do corpo, foi o motivo que mais levou os idosos a praticarem a automedicação. Este fato demonstra a importância da elevada frequência de quadro álgico em idosos e o uso recorrente de medicamentos para tratá-lo. $\underline{.2}$

Uma revisão sistemática ${ }^{23}$ demonstrou que o índice de automedicação em idosos é amplo e que as classes de medicamentos mais consumidas são os analgésicos, antipiréticos, anti-inflamatórios, de ação cardiovascular e os dietéticos. Os autores afirmam que há limitação quanto aos fatores que estão associados à prática da automedicação em idosos e que os estudos carecem de uma padronização do que é automedicação nesses indivíduos. Em nosso estudo, os idosos demonstram que são usuários de medicação de maneira contínua e que, além disso, não procuraram informações sobre os medicamentos que utilizaram quando praticaram a automedicação e que fazem uso de medicamentos que não são receitados pelo médico. Este fato demonstra que a prática da automedicação ocorre como busca ao alívio dos sintomas e é uma alternativa menos onerosa para reduzir os custos dos serviços de saúde. ${ }^{24}$

O estudo apresentou como limitação a não validação do instrumento para identificação do perfil de automedicação em idosos na atenção básica à saúde, além da amostra pequena. 


\section{Conclusão}

O estudo permitiu descrever a prática da automedicação em idosos atendidos na atenção básica, além dos motivos mais recorrentes, como quadro álgico e gripe/resfriado, que os levam a recorrerem a tal prática, bem como as situações cotidianas, como orientação farmacêutica e dificuldade de acesso ao profissional médico, que facilitam a automedicação.

Os idosos em sua maioria referiram que não receberam informações prévias sobre os riscos que tal prática proporciona, já confundiram os horários das medicações e já fizeram uso de medicações que não foram receitados pelo médico; e os anti-hipertensivos e antidiabéticos foram as classes de medicamentos mais consumidas.

Faz-se necessário que as medidas de educação em saúde sejam implementadas de maneira a sanar o déficit de informação sobre os riscos que a automedicação pode desencadear, além de facilitar o acesso ao profissional médico e alinhar as informações repassadas aos idosos junto com os farmacêuticos.

Os dados da presente pesquisa refletem sobre o perfil de uma pequena amostra de um município de pequeno porte, ainda que seus dados não possam ser extrapolados para a população em geral, muitos pontos observados são semelhantes aos de estudos anteriores e servem de base para futuras pesquisas.

\section{Agradecimentos}

Os pesquisadores agradecem a disponibilidade da SMS de Cocal-PI em realizar a pesquisa nas dependências da ESF Mutirão.

\section{Contribuições dos autores}

Silva TCA participou da concepção, planejamento, coleta e análise de dados, escrita e redação do manuscrito. Júnior FCCM participou da concepção do artigo. Silva JCA participou da análise dos dados e escrita do artigo. Carvalho JS participou da coleta de dados. Ribeiro MDA participou do planejamento do manuscrito. Biângulo FA participou da análise dos dados.

\section{Conflitos de interesses}

Nenhum conflito financeiro, legal ou político envolvendo terceiros (governo, empresas e fundações privadas, etc.) foi declarado para nenhum aspecto do trabalho submetido (incluindo, mas não se limitando a subvenções e financiamentos, participação em conselho consultivo, desenho de estudo, preparação de manuscrito, análise estatística, etc.).

\section{Referências}

1. Instituto Brasileiro de Geografia e Estatística. Perfil dos idosos responsáveis pelos domicílios no Brasil 2000 [Internet]. Rio de Janeiro: IBGE; 2002. Disponível em: https://biblioteca.ibge.gov.br/ index.php/biblioteca-catalogo?id=2929\&view=detalhes

2. Instituto Brasileiro de Geografia e Estatística. Pesquisa nacional por amostra de domicílios: síntese de indicadores 2015 [Internet]. $2^{\text {a }}$ ed. Rio de Janeiro: IBGE; 2016. Disponível em: https://biblioteca.ibge.gov.br/visualizacao/livros/liv98887.pdf

3. Amorim DNP, Chiarello MD, Vianna LG, Moraes CF, Vilaça KHC. Internações por condições sensíveis à atenção primária de idosos no Brasil, 2003 a 2012. Rev Enferm UFPE on line [Internet]. 2017;11(2):576-83. Disponível em: https://periodicos.ufpe.br/ revistas/revistaenfermagem/article/view/11976

4. Quinalha JV, Correr CJ. Instrumentos para avaliação da farmacoterapia do idoso: uma revisão. Rev Bras Geriatr Gerontol. 2010;13(3):487-99. https://doi.org/10.1590/S1809$\underline{98232010000300014}$

5. Gorzoni ML, Fabbri RMA, Pires SL. Medicamentos potencialmente inapropriados para idosos. Rev Assoc Med Bras. 2012;58(4):442-6. https://doi.org/10.1590/S010442302012000400014

6. Vernizi MD, Silva LL. A prática de automedicação em adultos e idosos: uma revisão de literatura. Rev Saúde e Desenvolvimento [Internet]. 2016;10(5):55-72. Disponível em: https://www.revistasuninter.com/revistasaude/index.php/ saudeDesenvolvimento/article/view/579

7. Lutz BH, Miranda VIA, Bertoldi AD. Inadequação do uso de medicamentos entre idosos em Pelotas, RS. Rev Saude Publica. 2017;51:52. https://doi.org/10.1590/s1518-8787.2017051006556

8. Sá M, Barros J. Automedicação em idosos na cidade de Salgueiro-PE. Rev Bras Epidemiol, 2007;10(1):75-85. http://dx.doi. org/10.1590/S1415-790X2007000100009 
9. Costa CMFN, Silveira MR, Acurcio FA, Guerra Junior AA, Guibu IA, Costa KS, et al. Utilização de medicamentos pelos usuários da atenção primária do sistema único de saúde. Rev de Saúde Pública, 2017; 51(supl. 2):18s. https://doi.org/10.11606/S15188787.2017051007144

10. Pereira FGF, Araújo MJP, Pereira CR, Nascimento DS, Galiza FT, Benício CDAV. Automedicação em idosos ativos. Rev enferm UFPE on line. 2017;11(12):4019-28. https://doi.org/10.5205/1981-8963v11i12a22289p4919-4928-2017

11. Meneguci J, Santos DAT, Silva RB, Santos RG, Sasaki JE, Tribess $\mathrm{S}$, et al. Comportamento sedentário: conceito, implicações fisiológicas e os procedimentos de avaliação. Motricidade. 2015;11(1):160-74. https://doi.org/10.6063/motricidade.3178

12. Monteiro SCM, Azevedo LS, Belfort IKP. Automedicação em idosos de um programa saúde da família, Brasil. Infarma. 2014;26(2):90-5. http://dx.doi.org/10.14450/2318-9312.v26. e2.a2014.pp90-95

13. Cardoso LO, Pinheiro SB, Mori B. Perfil da automedicação por idosos em uma Associação pública da cidade de Manaus - Amazonas. Scientia Amazonia [Internet]. 2018;7(3):38-44. Disponível em: http://scientia-amazonia.org/wp-content/ uploads/2018/08/v7-n3-cs38-cs44-2018.pdf

14. Silva BTF, Barros MLCMGR, Aquino DS, Vieira ACQM. O papel do farmacêutico no controle da automedicação em idosos. Boletim Informativo Geum [Internet]. 2017;8(3):18-31. Disponível em: https://revistas.ufpi.br/index.php/geum/article/view/5934

15. Santos TRA, Lima DM, Nakatani AYK, Pereira LV, Leal GS, Amaral RG. Consumo de medicamentos por idosos, Goiânia, Brasil. Rev Saúde Pública. 2013;47(1):94-103. https://doi. org/10.1590/S0034-89102013000100013

16. Sales AS, Sales MGS, Casotti CA. Perfil farmacoterapêutico e fatores associados à polifarmácia entre idosos de Aiquara, Bahia, em 2014. Epidemiol. Serv. Saúde. 2017;26(1):121-32. https://doi. org/10.5123/s1679-49742017000100013

17. Sardinha AHL, Silva CG, Sena LB, Mesquita LLS, Rodrigues JB, Silva KNR. Adesão dos idosos com doenças crônicas ao tratamento medicamentoso. Rev Pesq Saúde [Internet]. 2015;16(3):154-8. Disponível em: http://www. periodicoseletronicos.ufma.br/index.php/revistahuufma/article/ view/4513
18. Telles Filho PCP, Almeida AGP, Pinheiro MLP. Automedicação em idosos: um problema de saúde pública. Rev Enferm UERJ on line [Internet]. 2013;21(2):197-201. Disponível em: https://www.epublicacoes.uerj.br/index.php/enfermagemuerj/article/view/7107

19. Barroso R, Telles Filho PCP, Pinheiro MCP, Bodevan EC, Pereira Júnior AC, Cambraia RP. Automedicação em idosos de estratégias de saúde da família. Rev Enferm UFPE on line. 2017;11(suppl. 2):890-7. Disponível em: https://periodicos.ufpe.br/revistas/ revistaenfermagem/article/download/13457/16145

20. Bortolon PC, Medeiros EFF, Naves JOS, Karnikowski MGO, Nóbrega OT. Análise do perfil da automedicação em mulheres idosas brasileiras. Ciênc saúde coletiva. 2008;13(4):1219-26. https://doi.org/10.1590/S1413-81232008000400018

21. Oliveira SBV, Barroso SCC, Bicalho MAC, Reis AMM. Perfil de medicamentos utilizados por automedicação por idosos atendidos em centro de referência. Einstein. 2018;16(4):eAO4372. https://doi.org/10.31744/einstein_journal/2018a04372

22. Presa AA, Alvarez BR, Mazon J, Daminelli SA. Avaliação da capacidade funcional de idosas com artralgia praticantes de hidroginástica e cadastradas no programa de atenção multidisciplinar à saúde do idoso. Res., Soc. De [Internet]. 2020;9(10): e6129108972.Disponível em: https://redib.org/ Record/oai_articulo3006299-avalia\%C3\%A7\%C3\%A3o-dacapacidade-funcional-de-idosas-com-artralgia-praticantesde-hidrogin\%C3\%A1 stica-e-cadastradas-programa-deaten\%C3\%A7\%C3\%A3o-multidisciplinar-\%C3\%A0-sa\%C3\%BAdedo-idoso

23. Jerez-Roig J, Medeiros LF, Silva VA, Bezerra CL, Cavalcante LA, Piuvezam G, et al. Prevalence of self-medication and associated factors in an elderly population: a systematic review. Drugs Aging. 2014;31(12):883-96. https://doi.org/10.1007/s40266-014-0217-x

24. Oliveira MA, Francisco PMSB, Costa KS, Barros MBA. Automedicação em idosos residentes em Campinas, São Paulo, Brasil: prevalência e fatores associados. Cad Saúde Pública. 2012;28(2):335-45. https://doi.org/10.1590/S0102311X2012000200012 